\title{
Selective Extraction and Quantification of Glutathione using Maleimide-Presenting Gold Nanoparticles
}

\author{
Hongseok Oh, Jeongwook Lee, and Woon-Seok Yeo* \\ Department of Bioscience and Biotechnology, Bio/Molecular Informatics Center, Konkuk University, Seoul 143-701, Korea \\ *E-mail: wsyeo@konkuk.ac.kr
}

Received June 23, 2014, Accepted June 30, 2014

\begin{abstract}
In this paper, we describe a new method for the selective extraction and quantification of glutathione (GSH) using matrix-assisted laser desorption/ionization time-of-flight mass spectrometry (MALDI-TOF MS) and maleimide-presenting gold nanoparticles (Mal-AuNPs). Our strategy utilizes the Michael addition to selectively extract GSH, from chosen samples, onto the maleimide of Mal-AuNPs. After the extraction step, the GSH bound to the AuNPs was analyzed by MALDI-TOF MS in the presence of an internal standard which was prepared by reacting Mal-AuNPs with isotope-labeled GSH (GSH*). The GSH* has the same structure as GSH but a higher molecular weight, and therefore, enables absolute quantification of GSH by comparing the mass signal intensities of the GSH- and GSH*-conjugated alkanethiols. Our strategy was verified by analyzing GSHspiked fetal bovine serum and NIH 3 T3 cells.
\end{abstract}

Key Words : Glutathione, Quantification, Gold Nanoparticles, MALDI-TOF MS, Internal standard

\section{Introduction}

Glutathione (GSH), a thiol-containing tripeptide consisting of glycine, cysteine, and $\gamma$-glutamate, is the most abundant non-protein thiol present in living organisms. GSH plays important roles in various cellular activities including redox homeostasis as a major endogenous antioxidant, ${ }^{1}$ xenobiotic metabolism, ${ }^{2}$ and numerous signaling processes. ${ }^{3}$ In particular, in cellular redox homeostasis, GSH modulates the oxidative stress, which is associated with a number of diseases. ${ }^{4}$ In this respect, a GSH imbalance is observed in a wide range of pathologies including cancers, ${ }^{5}$ neurodegenerative disorders, ${ }^{6}$ cystic fibrosis, ${ }^{7}$ pulmonary disease, ${ }^{8}$ emphysema, ${ }^{9}$ and aging. ${ }^{10}$ Therefore, GSH detection and quantification is clinically important, and various methods to achieve this, including high performance liquid chromatography, ${ }^{11}$ capillary electrophoresis, ${ }^{12}$ fluorescence, ${ }^{13} \mathrm{UV} /$ visible spectrophotometry, ${ }^{14}$ and electrochemistry, ${ }^{15}$ have been reported. These methods often exploited the thiol functionality of GSH for its derivatization and subsequent detection/quantification. As a typical example, Ellman's reagent $\left(5,5^{\prime}\right.$-dithiobis(2-nitrobenzoic acid) or DTNB) is commonly used for the colorimetric measurement of biothiols by way of an absorbance enhancement due to a specific reaction between it and thiol groups. ${ }^{16}$ However, it is difficult to discriminate between GSH and other biothiols with similar chemical reactivity such as cysteine and homocyteine, using these methods.

In this respect, mass spectrometry is an ideal technique for the analysis of biomolecules with similar reactivity and even with structural similarity, because it can provide direct information about target molecules by measuring molecular weights. In particular, matrix-assisted laser desorption/ionization time-of-flight mass spectrometry (MALDI-TOF MS), a laser-based soft ionization technique, is widely used for the analysis of biomolecules, such as nucleic acids, peptides, carbohydrates, proteins, and lipids because of simple operating procedure, less fragmentation, and remarkable detection accuracy and sensitivity over a wide mass range, even in complex samples. ${ }^{17}$

Furthermore, MALDI-TOF MS is compatible with various nanomaterials, particularly, gold nanoparticles (AuNPs)

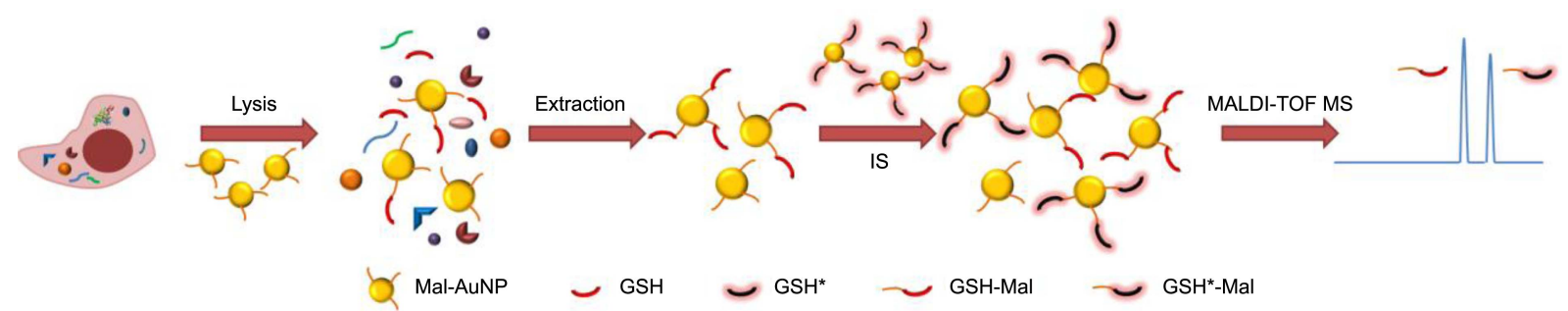

Scheme 1. A schematic diagram of the selective extraction and quantification of GSH present in cells. The cell lysate is incubated with Mal-AuNPs, onto which GSH is selectively extracted by means of Michael addition. The resulting GSH-conjugated AuNPs are analyzed by MALDI-TOF MS in the presence of IS which contains GSH*, isotope-labeled GSH. The comparison of the peak intensities of GSHconjugate alkanethiol and $\mathrm{GSH}^{*}$-conjugate alkanethiol allows absolute quantification of GSH in the cell lysate. 
because of their multifold advantages over other materials such as their high molar absorptivity, strong affinity toward thiol containing molecules and facile surface modifications, and easy fabrication of different sizes and shapes. ${ }^{18}$

Previous studies utilizing AuNPs and MALDI-TOF MS for GSH analysis have harnessed the specific gold-thiol interaction to directly extract GSH onto AuNPs. ${ }^{19}$

In these studies, however, quantitative analysis of GSH is not practical because MALDI-TOF MS is not inherently quantitative owing to poor shot-to-shot and sample-tosample reproducibility. ${ }^{20}$

Furthermore, consistent and stable GSH adsorption onto AuNPs may not be expected and oxidized GSH, disulfide form of GSH (GSSG), could also be adsorbed onto the AuNP surface.

As such, here, we report a method that addresses the above issues, the non-quantitative nature of MALDI-TOF MS and inconsistent GSH adsorption onto AuNPs, and therefore, enables quantification of GSH, by introducing an internal standard (IS) ${ }^{21}$ and using a structurally well-defined AuNP surface. An internal standard was designed to have the same molecular behavior as but a different molecular weight to the analyte in MALDI-TOF MS analysis such that the direct comparison of MS signal intensities between the analyte and IS would reflect the real amount of GSH present, and therefore, allow its absolute quantification. In addition, AuNPs that are pre-decorated with a structurally well-defined monolayer assure selective and consistent GSH immobilization.

Scheme 1 shows the schematic diagram for the selective extraction and quantification of GSH in cells. The cell lysate is incubated with maleimide-presenting AuNPs (Mal-AuNPs) prepared by an amide coupling reaction with the acidpresenting monolayer, which has been initially and uniformly formed on the AuNP surface. Michael addition between maleimides and thiols has been well characterized and widely used for the immobilization of thiol-containing biomolecules on the surface of various materials. The reaction proceeds selectively, rapidly, and essentially quantitatively over a wide $\mathrm{pH}$ range without the need for additional reagents. ${ }^{22}$ Therefore, GSH can be consistently and stably extracted onto the Mal-AuNPs by Michael addition between the GSH thiol and maleimide on the AuNPs. The resulting GSH-conjugated AuNPs are analyzed by MALDI-TOF MS in the presence of IS which contains isotope-labeled GSH $\left(\mathrm{GSH}^{*}\right)$ constructed from a glycine containing two carbons $\left({ }^{13} \mathrm{C}\right)$ and one nitrogen $\left({ }^{15} \mathrm{~N}\right)$ isotopes. GSH* is expected to behave identically to GSH in MALDI-TOF MS analysis and the comparison of mass peak intensity between GSHconjugated alkanethiol and $\mathrm{GSH}^{*}$-conjugated alkanethiol allows absolute quantification of GSH in the cell lysate.

\section{Experimental}

Materials. AuNPs (40 nm in diameter) were prepared using a method reported by Schwartzberg et al. ${ }^{23}$ For the preparation of tri(ethylene glycol)-terminated alkanethiol, carboxylic acid-terminated alkanethiol, and $\mathrm{N}$-aminoethyl maleimide, see the report by Houseman et al. and references therein. ${ }^{24}$ Sodium borohydride, 1-ethyl-3-(3-dimethylaminopropyl) carbodiimide hydrochloride (EDC), dimethyl sulfoxide (DMSO), glutathione (GSH), 2',4',6'-trihydroxyacetophenone monohydrate (THAP), L-sulforaphane (SFN), Lbuthionine sulfoximine (BSO), citric acid trisodium salt, and ammonium citrate were purchased from Sigma-Aldrich (St. Louis, MO, USA). Hydrogen tetrachloroaurate (III) hydrate was purchased from Kojima Chemicals Co., Ltd. (Sayama, Japan). Absolute ethanol ( $\geq 99.9 \%$ ) was purchased from Merck (Darmstadt, Germany). Acetonitrile (ACN) was purchased from Junsei Chemical Co., Ltd. (Tokyo, Japan). Isotope-labeled GSH (glycine residue labeled, ${ }^{15} \mathrm{~N}, 2 \times{ }^{13} \mathrm{C}$ ) was purchased from Cambridge Isotope Laboratories, Inc. (Andover, MA, USA). Dulbecco's modified eagle's medium (DMEM), phosphate-buffered saline (PBS), and fetal bovine serum (FBS) were purchased from WelGENE Inc. (Seoul, Korea).

Preparation of Acid-presenting AuNPs. AuNPs ( $1 \mathrm{~mL}$, $1.1 \mathrm{nM})$ were washed three times with distilled water by centrifugation (16000 g, $3 \mathrm{~min}$ ) and incubated with a mixed solution of tri(ethylene glycol)-terminated alkanethiol (100 $\mu \mathrm{M}$ in absolute ethanol) and carboxylic acid-terminated alkanethiol $(100 \mu \mathrm{M}$ in absolute ethanol) in a ratio of 7:3 for 12 h. The resulting AuNPs were washed three times with absolute ethanol by centrifugation (16000 g, $3 \mathrm{~min}$ ), suspended in $1 \mathrm{~mL}$ of absolute ethanol, and stored at $4{ }^{\circ} \mathrm{C}$.

Preparation of Mal-AuNPs. Acid-presenting AuNPs (1 $\mathrm{mL}, 1.1 \mathrm{nM}$ in absolute ethanol) were centrifuged (16000 g, $3 \mathrm{~min})$, treated with $\mathrm{N}$-aminoethyl maleimide $(300 \mu \mathrm{L}, 14$ $\mathrm{mg} / \mathrm{mL}$ in DMSO) and EDC (300 $\mu \mathrm{L}, 20 \mathrm{mg} / \mathrm{mL}$ in DMSO) for $1 \mathrm{~h}$, and washed with water and absolute ethanol by centrifugation (16000 g, $3 \mathrm{~min}$ ).

Construction of the Calibration Curve. Mal-AuNPs $(100 \mu \mathrm{L}, 1.1 \mathrm{nM}$ in absolute ethanol) were centrifuged (16000 $\mathrm{g}, 3 \mathrm{~min}$ ) and incubated with $100 \mu \mathrm{L}$ of GSH at various concentrations ranging from $1 \mu \mathrm{M}$ to $50 \mu \mathrm{M}$ in PBS for $2 \mathrm{~h}$. The mixture was washed with water and absolute ethanol by centrifugation (16000 g, $3 \mathrm{~min}$ ). The resulting GSH-conjugated AuNPs were suspended in $10 \mu \mathrm{L}$ of ammonium citrate $(0.5 \mathrm{mM}, \mathrm{pH} 4)$ and $3 \mu \mathrm{L}$ of this suspension was then mixed with $3 \mu \mathrm{L}$ of IS (GSH*-conjugated AuNPs, $1.1 \mathrm{nM}$ ) in ammonium citrate $(0.5 \mathrm{mM}, \mathrm{pH} 4)$. The mixture was analyzed by MALDI-TOF MS.

GSH Recovery from FBS. FBS was centrifuged (14000 g, $20 \mathrm{~min})$, diluted with PBS to various concentrations ranging from $1 \%$ to $20 \%(\mathrm{v} / \mathrm{v})$, and spiked with GSH (5 $\mu \mathrm{M}$ or $10 \mu \mathrm{M})$. Mal-AuNPs $(100 \mu \mathrm{L}, 1.1 \mathrm{nM}$ in absolute ethanol) was centrifuged (16000 g, $3 \mathrm{~min}$ ), incubated with $100 \mu \mathrm{L}$ of the GSH-spiked FBS for $2 \mathrm{~h}$, and washed with PBS, water, and ethanol by centrifugation (16000 g, $3 \mathrm{~min})$. The resulting AuNPs were suspended in $10 \mu \mathrm{L}$ of ammonium citrate $(0.5 \mathrm{mM}, \mathrm{pH} 4)$, and $3 \mu \mathrm{L}$ of this suspension was mixed with $3 \mu \mathrm{L}$ of IS in ammonium citrate $(0.5 \mathrm{mM}, \mathrm{pH} 4)$ and analyzed by MALDI-TOF MS.

Extraction and Quantification of GSH in NIH 3 T3 Cells. NIH 3 T3 cells were cultured in DMEM containing D- 
glucose $(4.5 \mathrm{~g} / \mathrm{L})$ supplemented with $10 \% \mathrm{FBS}$, penicillin (100 units $/ \mathrm{mL})$, and streptomycin $(100 \mathrm{~g} / \mathrm{mL})$. For inducing or suppressing intracellular GSH, cell culture media were exchanged with fresh media which contained either $15 \mu \mathrm{M}$ SFN (GSH inducer) or $50 \mu \mathrm{M}$ BSO (GSH suppressor). As controls, cells were also treated with media containing $1 \mathrm{mM}$ GSH or were left untreated. After incubation with SFN and GSH for $24 \mathrm{~h}$, and with BSO for $6 \mathrm{~h}$, cells $\left(\sim 2 \times 10^{6}\right)$ were collected by trypsinization and washed twice with PBS by centrifugation ( $250 \mathrm{~g}, 3 \mathrm{~min}$ ). The cells were subsequently suspended in PBS $(100 \mu \mathrm{L})$, lysed by sonication, and centrifuged (14000 g, $20 \mathrm{~min})$. The supernatant was collected and diluted with PBS (1:9, v/v). Mal-AuNPs $(100 \mu \mathrm{L}, 1.1$ $\mathrm{nM}$ in absolute ethanol) was centrifuged (16000 g, $3 \mathrm{~min})$, incubated with $100 \mu \mathrm{L}$ of the lysate for $2 \mathrm{~h}$, and washed successively with PBS, water, and absolute ethanol by centrifugation (16000 g, $3 \mathrm{~min}$ ). The resulting AuNPs were suspended in $10 \mu \mathrm{L}$ of ammonium citrate $(0.5 \mathrm{mM}, \mathrm{pH} 4)$, and $3 \mu \mathrm{L}$ of this suspension was mixed with $3 \mu \mathrm{L}$ of IS in ammonium citrate $(0.5 \mathrm{mM}, \mathrm{pH} 4)$ and analyzed by MALDITOF MS.

MALDI-TOF MS Analysis. Mass analysis was performed using an Autoflex III MALDI-TOF mass spectrometer (Bruker Daltonics, Germany) equipped with a smartbeam laser as an ionization source. All of the spectra were acquired with a $19 \mathrm{kV}$ accelerating voltage, a $100 \mathrm{~Hz}$ repetition rate, in positive mode with an average of $\sim 1000$ shots using a THAP ( $3 \mathrm{mg} / \mathrm{mL}$ in 1:1 (v/v) mixture of $\mathrm{ACN}$ and ammonium citrate) as a matrix.

\section{Results and Discussion}

First, we verified the selective conjugation of GSH and
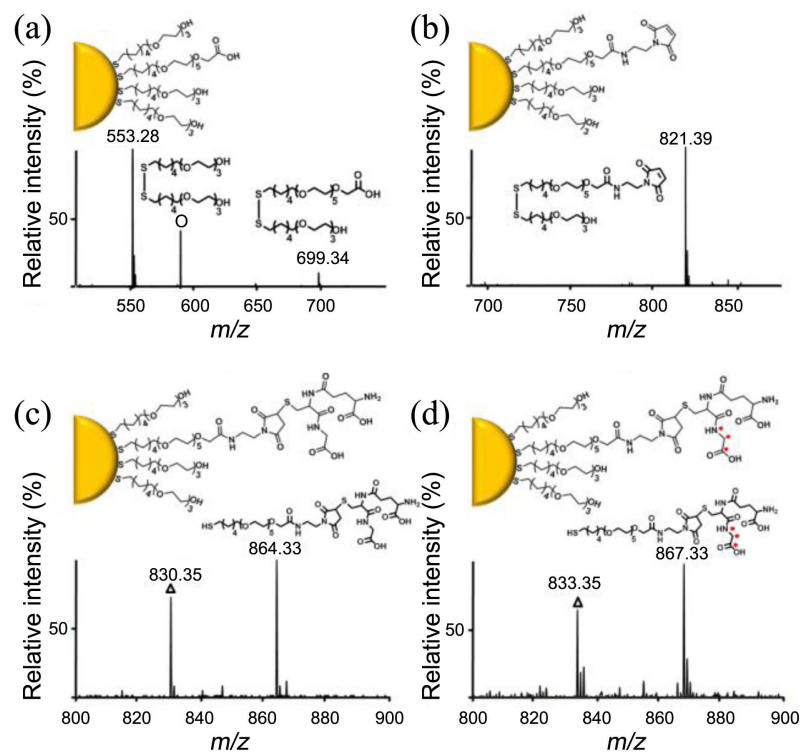

Figure 1. Verification of GSH- and GSH*-conjugated AuNPs using MALDI-TOF MS. MS spectra of (a) acid-presenting AuNPs, (b) maleimide-presenting AuNPs, (c) GSH-conjugated AuNPs, and (d) GSH*-conjugated AuNPs. (O: Peaks from AuNPs, and $\left.\triangle:\left[\mathrm{M}-\mathrm{H}_{2} \mathrm{~S}+\mathrm{Na}\right]^{+}\right)$.
$\mathrm{GSH}^{*}$ to the Mal-AuNPs using MALDI-TOF MS. Figure 1 shows the structures of monolayers on AuNPs and the chemical conversions used in this study. Acid-presenting AuNPs were prepared with a tri(ethylene glycol)-terminated alkanethiol and a carboxylic acid-terminated alkanethiol in a ratio of 7:3. Monolayer composition was confirmed by the presence of peaks at $\mathrm{m} / z 553.3[\mathrm{M}+\mathrm{Na}]^{+}$and $\mathrm{m} / \mathrm{z} 699.3$ $[\mathrm{M}+\mathrm{Na}]^{+}$corresponding to tri(ethylene glycol)-containing disulfide and acid-containing disulfide, respectively (Figure 1(a)). The tri(ethylene glycol) group provides inertness to the AuNPs by preventing non-specific protein adsorption and direct GSH adsorption onto the AuNP surface which may result in unpredictable analysis. The acid-presenting AuNPs were then treated with EDC and $N$-aminoethyl maleimide. MS analysis gave a peak at $m / z 821.4[\mathrm{M}+\mathrm{Na}]^{+}$ indicating that the maleimide functionality was successfully incorporated to produce Mal-AuNPs (Figure 1(b)). The resulting Mal-AuNPs were incubated with GSH and GSH* and analyzed by MALDI-TOF MS. As shown in Figure 1(c) and Figure 1(d), after incubation, the peak at $m / z 821.4$ was absent and replaced with peaks at $m / z 864.3[\mathrm{M}+\mathrm{Na}]^{+}$and $m / z 867.3[\mathrm{M}+\mathrm{Na}]^{+}$corresponding to $\mathrm{GSH}-$ and $\mathrm{GSH}^{*}-$ conjugated alkanethiols, respectively. These results indicate that GSH and GSH* reacted effectively with the Mal-AuNPs by means of Michael addition and the reaction had proceeded essentially quantitatively. Note that the peaks at $\mathrm{m} / \mathrm{z}$ 830.4 and 833.4 resulted from an abstraction of hydrogen sulfide $\left(\left[\mathrm{M}-\mathrm{H}_{2} \mathrm{~S}+\mathrm{Na}\right]^{+}\right)$from the $\mathrm{GSH}-$ and $\mathrm{GSH}^{*}$-conjugated alkanethiols.

Subsequently, we examined the feasibility of our strategy for GSH quantification. Mal-AuNPs were incubated with $\mathrm{GSH}$ at various concentrations ranging from $1 \mu \mathrm{M}$ to 50 $\mu \mathrm{M}$. After washing with water and ethanol by centrifugation,

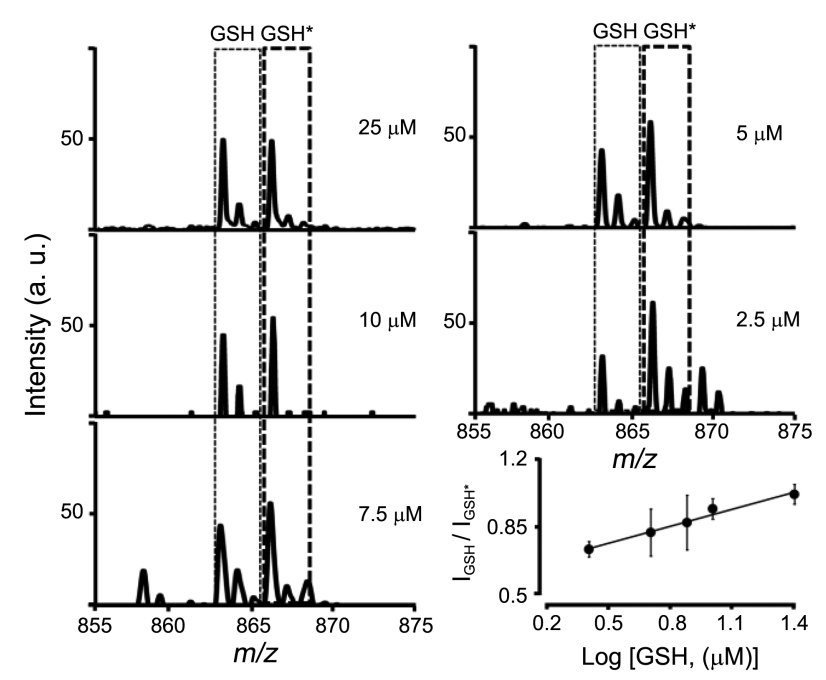

Figure 2. Concentration dependence of GSH detection. (a) Representative MALDI-TOF MS spectra of GSH-conjugated AuNPs at various GSH concentration in the presence of a fixed amount of IS. (b) A calibration curve constructed by a linear regression of the peak intensity ratio between GSH- and $\mathrm{GSH}^{*}$-conjugated alkanethiols versus GSH concentration. The standard deviations were obtained from at least three independent experiments. 
the resulting GSH-conjugated AuNPs were mixed with IS (GSH*-conjugated AuNPs) and analyzed by MALDI-TOF MS. The mixtures were suspended in ammonium citrate solution $(0.5 \mathrm{mM}, \mathrm{pH} 4)$ that is known to minimize the formation of multiple sodium adducts. ${ }^{21 a}$ Figure 2 shows the representative mass spectra of the mixtures at various GSH concentrations and the calibration curve constructed by a linear regression of the peak intensity ratio of GSH- and GSH*-conjugated alkanethiols versus GSH concentration. As expected, both the GSH- and $\mathrm{GSH}^{*}$-conjugated alkanethiols were distinctly observed and the peak intensities of GSHconjugated alkanethiols gradually increased with increasing GSH concentration The calibration curve is linear in the GSH concentration range from $2.5 \mu \mathrm{M}$ to $25 \mu \mathrm{M}\left(\mathrm{R}^{2}=\right.$ 0.9781). MS analysis showed that detection limit of the method was $1 \mu \mathrm{M}(\mathrm{S} / \mathrm{N} \geq 3)$, and that GSH concentrations over $25 \mu \mathrm{M}$ were not in the linear range. The dynamic range of our method makes it suitable for GSH detection because it is in good accordance with the physiological GSH concentration range. ${ }^{25}$

After confirming the feasibility of our strategy, we investigated its capability to quantify the amount of GSH in complex samples. This was performed using fetal bovine serum (FBS, $1-20 \%$ in PBS) spiked with GSH at concentrations of either $5 \mu \mathrm{M}$ or $10 \mu \mathrm{M}$. The Mal-AuNPs were incubated with the GSH-spiked FBS, washed with PBS, water and ethanol by centrifugation, suspended in ammonium citrate solution, and analyzed by MALDI-TOF MS in the presence of IS. As shown in Table 1, recoveries from $93 \%$ to $108 \%$ of a known amount of GSH added to FBS were obtained. This result clearly indicates the feasibility and the applicability of our method to complex samples.

Finally, we applied our system to monitor GSH concentration variations in cells in response to external stimulants. NIH 3T3 cells were incubated with either GSH synthesis inducer or a suppressor, and the change in GSH concentration was investigated. L-Sulforaphane (SFN), which has been known to prevent oxidative stress-induced cell death by increasing intracellular GSH concentration, was used as an inducer. ${ }^{26} \mathrm{~L}$-Buthionine sulfoximine (BSO), which decreases the GSH concentration by irreversibly inhibiting the activity of $\gamma$-glutamylcysteine synthetase, was used as a suppressor. ${ }^{27}$ We also examined the addition of external GSH that can affect the intracellular GSH concentration by being internalized

Table 1. GSH recovery from FBS

\begin{tabular}{cccc}
\hline FBS conc. (v/v) & Spiked GSH & Measured GSH & Recovery \\
\hline $1 \%$ & $5.0 \mu \mathrm{M}$ & $5.2 \mu \mathrm{M}$ & $105 \%$ \\
$1 \%$ & $10.0 \mu \mathrm{M}$ & $9.6 \mu \mathrm{M}$ & $96 \%$ \\
$5 \%$ & $5.0 \mu \mathrm{M}$ & $5.0 \mu \mathrm{M}$ & $100 \%$ \\
$5 \%$ & $10.0 \mu \mathrm{M}$ & $10.5 \mu \mathrm{M}$ & $105 \%$ \\
$10 \%$ & $5.0 \mu \mathrm{M}$ & $5.2 \mu \mathrm{M}$ & $103 \%$ \\
$10 \%$ & $10.0 \mu \mathrm{M}$ & $10.8 \mu \mathrm{M}$ & $108 \%$ \\
$20 \%$ & $5.0 \mu \mathrm{M}$ & $5.3 \mu \mathrm{M}$ & $105 \%$ \\
$20 \%$ & $10.0 \mu \mathrm{M}$ & $9.3 \mu \mathrm{M}$ & $93 \%$ \\
\hline
\end{tabular}

\begin{tabular}{cc}
\hline Regulator & $\begin{array}{c}\text { Measured GSH } \\
\text { (nmol/106 cells) }\end{array}$ \\
\hline Not treated & $5.26 \pm 0.80$ \\
SFN & $12.4 \pm 0.41$ \\
GSH & $10.4 \pm 1.61$ \\
BSO & $1.34 \pm 0.23$ \\
\hline
\end{tabular}

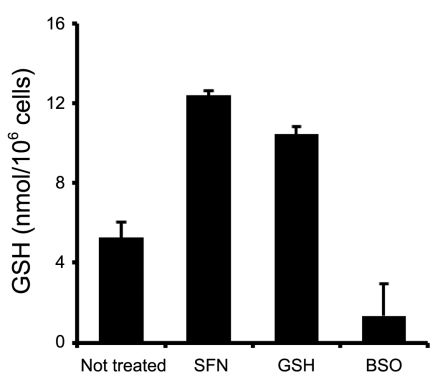

Figure 3. Quantification of GSH in NIH 3 T3 cells after the GSH regulator was treated with either L-sulforaphane (SFN) as an inducer or L-buthionine sulfoximine (BSO) as a suppressor. As controls, cells were treated with GSH or were left untreated.

into cells through the membrane transport system. ${ }^{28}$ After incubation with SFN $(10 \mu \mathrm{M})$, BSO $(50 \mu \mathrm{M})$, and GSH (1 $\mathrm{mM}$ ), the cells were lysed and centrifuged. The lysate was diluted with PBS (1:9, v/v) and incubated with Mal-AuNPs for $2 \mathrm{~h}$. After washing, the resulting AuNPs were suspended in ammonium citrate solution, and analyzed by MALDITOF MS in the presence of a known amount of IS. As shown in Figure 3, the GSH concentration of NIH 3T3 cells without treatment was $5.26 \pm 0.80 \mathrm{nmol} / 10^{6}$ cells. After treatment with SFN and GSH, the GSH concentration increased to $12.4 \pm 0.41 \mathrm{nmol} / 10^{6}$ cells and $10.4 \pm 1.61 \mathrm{nmol} / 10^{6}$ cells, respectively. On the contrary, treatment with $\mathrm{BSO}$ resulted in a GSH concentration of $1.34 \pm 0.23 \mathrm{nmol} / 10^{6}$ cells. These results are in good accordance with previous studies ${ }^{28 \mathrm{a}, 29}$ suggesting that the present method can be used practically for monitoring intracellular GSH concentration changes.

\section{Conclusion}

In summary, a simple strategy for the absolute quantification of GSH has been demonstrated by combining MALDI-TOF MS and AuNPs. The use of AuNPs coated with maleimide-presenting monolayers ensured consistent GSH immobilization while an IS which contains isotopelabeled GSH allowed for the quantification of GSH by comparing $\mathrm{m} / \mathrm{z}$ peak intensities. Our strategy was verified by the absolute quantification of GSH in two complex samples, FBS and cell lysate. The results strongly suggest that this strategy could be a general platform for the quantification of thiol-containing molecules and could be further extended to other biothiols.

Acknowledgments. This research was supported by the Basic Science Research Program (NRF-2013R1A1A2007272) and the Priority Research Centers Program (2009-0093824) through the National Research Foundation (NRF) of Korea funded by the Ministry of Education.

\section{References}

1. Lushchak, V. I. J. Amino Acids 2012, 2012, 736837.

2. (a) Fang, Y.-Z.; Yang, S.; Wu, G. Nutrition 2002, 18, 872. (b) Pastore, A.; Federici, G.; Bertini, E.; Piemonte, F. Clin. Chim. 
Acta 2003, 333, 19.

3. (a) Sies, H. Free Radical Bio. Med. 1999, 27, 916. (b) Fratelli, M.; Goodwin, L. O.; Ørom, U. A.; Lombardi, S.; Tonelli, R.; Mengozzi, M.; Ghezzi, P. Proc. Natl. Acad. Sci. USA 2005, 102, 13998.

4. Townsend, D. M.; Tew, K. D.; Tapiero, H. Biomed. Pharmacother. 2003, 57, 145 .

5. Cook, J. A.; Pass, H. I.; Iype, S. N.; Friedman, N.; DeGraff, W.; Russo, A.; Mitchell, J. B. Cancer Res. 1991, 51, 4287.

6. Chi, L.; Ke, Y.; Luo, C.; Gozal, D.; Liu, R. Neuroscience 2007, 144, 991.

7. (a) Roum, J. H.; Buhl, R.; McElvaney, N. G.; Borok, Z.; Crystal, R. G. J. Appl. Physiol. 1993, 75, 2419. (b) Hudson, V. M. Free Radical Bio. Med. 2001, 30, 1440.

8. Engelen, M. P. K. J.; Orozco-Levi, M.; Deutz, N. E. P.; Barreiro, E.; Hernández, N.; Wouters, E. F. M.; Gea, J.; Schols, A. M. W. J. Eur. Respir. J. 2004, 23, 545.

9. Engelen, M. P. K. J.; Schols, A. M. W. J.; Does, J. D.; Deutz, N. E. P.; Wouters, E. F. M. Am. J. Respir. Crit. Care Med. 2000, 161, 98.

10. Sastre, J.; Pallardó, F.; Viña, J. Age 1996, 19, 129.

11. (a) Jones, D. P.; Carlson, J. L.; Samiec, P. S.; Sternberg, P., Jr.; Mody, V. C., Jr.; Reed, R. L.; Brown, L. A. S. Clin. Chim. Acta 1998, 275, 175. (b) Wang, X.; Chi, D.; Song, D.; Su, G.; Li, L.; Shao, L. J. Chromatogr. Sci. 2012, 50, 119.

12. (a) Serru, V.; Baudin, B.; Ziegler, F.; David, J.-P.; Cals, M.-J.; Vaubourdolle, M.; Mario, N. Clin. Chem. 2001, 47, 1321. (b) Carru, C.; Zinellu, A.; Mario Pes, G.; Marongiu, G.; Tadolini, B.; Deiana, L. Electrophoresis 2002, 23, 1716.

13. (a) Lim, S.-Y.; Lee, S.; Park, S. B.; Kim, H.-J. Tetrahedon Lett. 2011, 52, 3902. (b) Guo, Y.; Wang, H.; Sun, Y.; Qu, B. Chem. Comm. 2012, 3221. (c) Gao, Y.; Li, Y.; Zou, X.; Huang, H.; Su, X. Anal. Chim. Acta 2012, 731, 68.

14. (a) Kim, G.-J.; Lee, K.; Kwon, H.; Kim, H.-J. Org. Lett. 2011, 13, 2799. (b) Xu, H.; Wang, Y.; Huang, X.; Li, Y.; Zhang, H.; Zhong, X. Analyst 2012, 137, 924.

15. (a) Kim, S.; Kim, J. I.; Park, H. R.; Kim, M. K.; Chong, Y.; Yeo, W.-S. Bull. Korean Chem. Soc. 2009, 30, 2574. (b) Harfield, J. C.; Batchelor-McAuley, C.; Compton, R. G. Analyst 2012, 137, 2285.

16. (a) Sanz-Alfayate, G.; Obeso, A.; Agapito, M. T.; González, C. J. Physiol. 2001, 537, 209. (b) Garcia, S. C.; Schott, K.; Charão, M.; Moro, A.; Bulcão, R.; Grotto, D.; Valentini, J.; Bohrer, D.; Cardoso, S.; Pomblum, V. Biomed. Chromatogr. 2008, 22, 460.

17. (a) Tanaka, K.; Waki, H.; Ido, Y.; Akita, S.; Yoshida, Y.; Yoshida, T.; Matsuo, T. Rapid Commun. Mass Spectrom. 1988, 2, 151. (b) Karas, M.; Hillenkamp, F. Anal. Chem. 1988, 60, 2299. (c) Kim, Y. J.; Freas, A.; Fenselau, C. Anal. Chem. 2001, 73, 1544. (d) Marvin, L. F.; Roberts, M. A.; Fay, L. B. Clin. Chim. Acta 2003, 337, 11. (e) Rujoi, M.; Estrada, R.; Yappert, M. C. Anal. Chem.
2004, 76, 1657. (f) Jurinke, C.; Oeth, P.; Boom, D. Mol. Biotechnol. 2004, 26, 147. (g) Selman, M. H. J.; Hoffmann, M.; Zauner, G.; McDonnell, L. A.; Balog, C. I. A.; Rapp, E.; Deelder, A. M.; Wuhrer, M. Proteomics 2012, 12, 1337.

18. (a) Watanabe, T.; Kawasaki, H.; Yonezawa, T.; Arakawa, R. J. Mass Spectrom. 2008, 43, 1063. (b) Lim, A. Y.; Ma, J.; Boey, Y. C. F. Adv. Mater. 2012, 24, 4211. (c) Lee, J.; Lee, J.; Chung, T. D.; Yeo, W.-S. Anal. Chim. Acta 2012, 736, 1.

19. (a) Lee, J.; Lee, J.; Oh, H.; Mok, H.; Yeo, W. S. Bull. Korean Chem. Soc. 2012, 33, 3076. (b) Wan, D.; Gao, M.; Wang, Y.; Zhang, P.; Zhang, X. J. Sep. Sci. 2013, 36, 629.

20. (a) Albrethsen, J. Clin. Chem. 2007, 53, 852. (b) Szájli, E.; Fehér, T.; Medzihradszky, K. F. Mol. Cell Proteomics 2008, 7, 2410.

21. (a) Chiang, C.-K.; Lin, Y.-W.; Chen, W.-T.; Chang, H.-T. Nanomed. Nanotechnol. Biol. Med. 2010, 6, 530. (b) Hong, S.-H.; Kim, J. I.; Kang, H.; Yoon, S.; Kim, D.-E.; Jung, W.; Yeo, W.-S. Chem. Comm. 2014, 4831.

22. (a) Houseman, B. T.; Gawalt, E. S.; Mrksich, M. Langmuir 2002, 19, 1522. (b) Seo, H.; Choi, I.; Lee, J.; Kim, S.; Kim, D.-E.; Kim, S. K.; Yeo, W.-S. Chem. Eur. J. 2011, 17, 5804. (c) Lee, J.; Choi, I.; Yeo, W.-S. Chem. Eur. J. 2013, 19, 5609.

23. Schwartzberg, A. M.; Olson, T. Y.; Talley, C. E.; Zhang, J. Z. J. Phys. Chem. B 2006, 110, 19935.

24. Houseman, B. T.; Gawalt, E. S.; Mrksich, M. Langmuir 2003, 19, 1522 .

25. (a) Morrison, J. A.; Jacobsen, D. W.; Sprecher, D. L.; Robinson, K.; Khoury, P.; Daniels, S. R. Circulation 1999, 100, 2244. (b) Pastore, A.; Piemonte, F.; Locatelli, M.; Lo Russo, A.; Gaeta, L. M.; Tozzi, G.; Federici, G. Clin. Chem. 2001, 47, 1467.

26. (a) Tarozzi, A.; Morroni, F.; Merlicco, A.; Hrelia, S.; Angeloni, C.; Cantelli-Forti, G.; Hrelia, P. J. Neurochem. 2009, 111, 1161. (b) Bongard, R. D.; Krenz, G. S.; Gastonguay, A. J.; Williams, C. L.; Lindemer, B. J.; Merker, M. P. Free Radical Bio. Med. 2011, 50, 953.

27. Iantomasi, T.; Favilli, F.; Degl'Innocenti, D.; Vincenzini, M. T. BBA-Mol. Cell Res. 1999, 1452, 303.

28. (a) Favilli, F.; Catarzi, S.; Iantomasi, T.; Vincenzini, M. T. Mol. Cell Biol. Res. Commun. 2000, 4, 212. (b) Iantomasi, T.; Favilli, F.; Catarzi, S.; Giannoni, E.; Biagioni, C.; Vincenzini, M. IUBMB Life 2003, 55, 159.

29. (a) Rigacci, S.; Iantomasi, T.; Marraccini, P.; Berti, A.; Vincenzini, M. T.; Ramponi, G. Biochem. J. 1997, 324, 791. (b) Susanto, I.; Wright, S. E.; Lawson, R. S.; Williams, C. E.; Deneke, S. M. Am. J. Physiol. 1998, 274, L296. (c) Sun, X.; Mi, L.; Liu, J.; Song, L.; Chung, F.-L.; Gan, N. Toxicol. Appl. Pharmacol. 2011, 255, 9. (d) Akhtar, M. J.; Ahamed, M.; Fareed, M.; Alrokayan, S. A.; Kumar, S. J. Toxicol. Sci. 2012, 37, 139. 